\title{
Aluminum Silicate Nanotube Coating of Siloxane-Poly(lactic acid)-Vaterite Composite Fibermats for Bone Regeneration
}

\author{
Shuji Yamazaki, ${ }^{1}$ Hirotaka Maeda, ${ }^{2}$ Akiko Obata, ${ }^{1}$ Keiichi Inukai, ${ }^{3}$ \\ Katsuya Kato, ${ }^{4}$ and Toshihiro Kasuga ${ }^{1}$ \\ ${ }^{1}$ Department of Frontier Materials, Nagoya Institute of Technology, Gokiso-cho, Showa-ku, Nagoya 466-8555, Japan \\ ${ }^{2}$ Center for Fostering Young and Innovative Researchers, Nagoya Institute of Technology, Gokiso-cho, Showa-ku, \\ Nagoya 466-8555, Japan \\ ${ }^{3}$ Ecological Ceramics Group, Materials Research Institute for Sustainable Development, National Institute of Advanced Industrial \\ Science and Technology, Anagahora Shimo-shidami, Moriyama-ku, Nagoya 463-8560, Japan \\ ${ }^{4}$ Bio-integration Processing Research Group, Advanced Manufacturing Research Institute, National Institute of Advanced Industrial \\ Science and Technology, Anagahora Shimo-shidami, Moriyama-ku, Nagoya 463-8560, Japan
}

Correspondence should be addressed to Toshihiro Kasuga, kasuga.toshihiro@nitech.ac.jp

Received 20 July 2012; Revised 16 November 2012; Accepted 26 November 2012

Academic Editor: Yong Yang

Copyright (c) 2012 Shuji Yamazaki et al. This is an open access article distributed under the Creative Commons Attribution License, which permits unrestricted use, distribution, and reproduction in any medium, provided the original work is properly cited.

\begin{abstract}
In our earlier work, a flexible fibermat consisting of a biodegradable composite with soluble silicate species, which has been reported to enhance bone formation, was prepared successfully using poly(L-lactic acid) and siloxane-containing calcium carbonate particles by electrospinning. The fibermat showed enhanced bone formation in an in vivo test. In the present work, to improve the hydrophilicity of skeletal fibers in a fibermat, they were coated with nanotubular aluminum silicate crystals, which have a hydrophilic surface that has excellent affinity to body fluids and a high surface area advantageous for pronounced protein adsorption. The nanotubes were coated easily on the fiber surface using an electrophoretic method. In a conventional contact angle test, a drop of water rapidly penetrated into the nanotube-coated fibermat. The culture test using murine osteoblast-like cells (MC3T3-E1) showed that the cell attachment to the nanotube-coated fibermat at an early stage after seeding was enhanced in comparison with that to the noncoated one. This approach may provide a new method of improving the surface of polymer-based biomaterials.
\end{abstract}

\section{Introduction}

Many types of scaffolds have been investigated for use in bone regeneration $[1,2]$, which serve as templates for cell interaction and the formation of a bone extracellular matrix (ECM) to provide structural support to newly formed tissues. Ceramics, such as hydroxyapatite (HA) and $\beta$-tricalcium phosphate $(\beta$-TCP), show good biocompatibility, but they are brittle and thus are difficult to form into various shapes. In contrast, synthetic biodegradable polymers are more easily shaped and show flexibility. However, they may elicit an inflammatory response in the host tissue because of the release of acidic degradation products [3]. Considering these problems, composites have been fabricated using biodegradable polymers coupled with calcium phosphate ceramics, whose excellent biocompatibility is established [4, 5].

The present authors have developed a novel composite consisting of poly(L-lactic acid) (PLLA), which is a biodegradable polymer that has already been applied clinically, and siloxane-containing vaterite $(\mathrm{SiV})$; the siloxane-PLLAvaterite hybrid-composite is denoted as $\mathrm{SiPVH}$, hereafter. It can release calcium and silicate ions, which have been reported to enhance the proliferation, differentiation, and mineralization of osteoblasts [6-8]. The cellular compatibility of SiPVH was tested using mouse osteoblast-like cells [9]. A film of SiPVH was prepared by dip-coating a coverglass with the composite solution. The film showed the release 
of calcium and silicate ions into the culture medium. Cell proliferation and mineralization on the composite film were enhanced in comparison with those on an undoped vateritePLA composite film, which showed no silicon release.

Three-dimensional scaffolds are common in biomaterials. Electrospinning is a process for forming fibermats with a high porosity and a high flexibility [10-12]. These fibermats are very promising for the regeneration of tissues and organs $[11,13]$ owing to the possibility of mimicking some structural features of natural ECM [14]. Numerous studies have shown the enhanced cellular attachment and proliferation on fibrous materials [15-17]. The present authors have successfully prepared a SiPVH fibermat by an electrospinning method and then performed in vivo experiments by placing the fibermat with $8 \mathrm{~mm}$ defects in the front midline of the calvaria of New Zealand rabbits $[18,19]$. They found enhanced bone formation on SiPVH fibermats. PLLA as a matrix phase in SiPVH is relatively hydrophobic. This is a property to be improved for the adhesion of bone-forming cells at the initial stage after implantation in the body.

A naturally occurring hydrated aluminum silicate, $(\mathrm{HO})_{3} \mathrm{Al}_{2} \mathrm{O}_{3} \mathrm{Si}(\mathrm{OH})$, which is called imogolite, has a nanotubular structure of $\sim 2.2 \mathrm{~nm}$ external and $\sim 1.0 \mathrm{~nm}$ internal diameters [20], with a length of up to several hundreds of micrometers. The imogolite nanotube (INT) is a hydrophilic material owing to numerous hydroxyl groups on its surface and has a high surface area, resulting in a high protein adsorption activity [21-23]. Ishikawa and coworkers reported that the amount of normalized protein per osteoblast-like cell (SaOS-2) cultured on INTs is two times larger than that cultured on a culture dish, and that the cells bond more strongly to INTs than to a culture dish [24, 25]. These results demonstrate the good biocompatibility between cells and INTs: thus, INTs may be useful for various biomedical applications.

In the present work, SiPVH fibermats were coated with INTs to improve their hydrophilicity to enhance cellular compatibility at the early stage after implantation.

\section{Materials and Methods}

2.1. Preparation of SiPVH Fibermats. SiV particles of $1 \mu \mathrm{m}$ diameter, containing $2.6 \mathrm{wt} \%$ silicon, were prepared by a carbonation method using methanol and $\gamma$-aminopropyltriethoxysilane (APTES; Momentive Performance Materials, Japan) as the silica precursor, as described in our previous works $[9,18,19]$. One hundred and fifty grams of $\mathrm{Ca}(\mathrm{OH})_{2}$, $60 \mathrm{~mL}$ of APTES and $2 \mathrm{~L}$ of methanol were mixed by bubbling a $\mathrm{CO}_{2}$ gas in the resulting mixture for $75 \mathrm{~min}$ at a rate of $2 \mathrm{~L} \cdot \mathrm{min}^{-1}$. The resulting slurry was dried at $110^{\circ} \mathrm{C}$, resulting in the formation of spherical SiV particles.

SiPVH was prepared by kneading PLLA (Mitsui Chemicals, LACIA; molecular weight: $\mathrm{Mw}=\sim 140 \mathrm{kDa}$ ) with $\mathrm{SiV}$ particles at $200^{\circ} \mathrm{C}$ for $10 \mathrm{~min}$ in a weight ratio of PLLA/SiV = $7 / 3$.

SiPVH was dissolved in chloroform for electrospinning to prepare $10 \mathrm{wt} \%$ PLLA solution. The fibermats were prepared by electrospinning at a voltage of $20 \mathrm{kV}$ using the conditions and compositions previously found to be optimal for preparing microfibers of $\sim 10 \mu \mathrm{m}$ diameter $[18,19]$. The flow rate of the chloroform solution was $50 \mu \mathrm{L} \cdot \mathrm{min}^{-1}$, and the distance between the nozzle and the collector was $150 \mathrm{~mm}$. The drum-shaped collector was rotated at $2 \mathrm{~m} \cdot \mathrm{min}^{-1}$, and the syringe filled with the SiPVH solution was traversed at $70-100 \mathrm{~mm} \cdot \mathrm{min}^{-1}$ from side to side.

2.2. Preparation of INT-Dispersed Aqueous Solution. INTs were synthesized, essentially following a method described by Suzuki et al. [26]. In $500 \mathrm{~mL}$ of distilled water (DW), $18.47 \mathrm{~g}$ of aluminum chloride $\left(\mathrm{AlCl}_{3} \cdot 6 \mathrm{H}_{2} \mathrm{O}\right.$; Wako Pure Chemicals) and $9.2 \mathrm{~g}$ of sodium silicate $\left(\mathrm{Na}_{4} \mathrm{SiO}_{4} \cdot n \mathrm{H}_{2} \mathrm{O}\right.$; Wako Pure Chemicals) were dissolved to yield a Si/Al molar ratio of 0.41 .

Sodium hydroxide (NaOH; Wako Pure Chemicals) aqueous solution of $1 \mathrm{M}$ was slowly added to the abovedescribed sodium silicate/aluminum chloride solution at a rate of $2 \mathrm{~mL} \cdot \mathrm{min}^{-1}$ until the $\mathrm{pH}$ of the solution reached 6.8. The sample was separated by centrifugation and the obtained precipitates were rinsed in DW with stirring. After the centrifugation-rinsing process was repeated three times, the resulting aluminum silicate precursors were dispersed again in $12 \mathrm{~L}$ of DW. Subsequently, the solution was acidified again by the addition of $12 \mathrm{~mL}$ of hydrochloric acid (HCl; Wako Pure Chemicals) with a concentration of $5 \mathrm{M}$ and then heated at $95^{\circ} \mathrm{C}$ for 1 or $4 \mathrm{~d}$ for INT formation. Consequently, the resulting INTs were dispersed in an aqueous solution with a concentration of $0.087 \mathrm{wt} \%$. The INTs were observed by atomic force microscopy (AFM) in the tapping mode. Figure 1 shows AFM images of the products after heating at $95^{\circ} \mathrm{C}$. The average lengths of the INTs obtained after the heating for 1 and $4 \mathrm{~d}$ were 220 and $570 \mathrm{~nm}$, respectively. They are denoted as $\mathrm{INT}_{220}$ and $\mathrm{INT}_{570}$, respectively, hereafter.

2.3. INT Coating of SiPVH Fibermats. The INT coating of SiPVH fibermats was achieved using an electrophoretic deposition (EPD) method.

Each fibermat was cut into $15 \mathrm{~mm} \times 15 \mathrm{~mm} \times 0.2 \mathrm{~mm}$ dimensions with scissors and then placed on $15 \mathrm{~mm} \times$ $60 \mathrm{~mm} \times 0.25 \mathrm{~mm}$ aluminum foil. One hundred microliters of ethanol were dropped on the foil to fix each fibermat on the cathode electrode.

The objective of the present work is to prepare a hydrophilic coating with an extremely thin layer of INTs on skeletal fibers. Our preliminary work showed the EPD conditions for the preparation. The point of zero charge (PZC) in an INTcontaining solution has been reported to be $\mathrm{pH} 6.0$ [27]. The INT-dispersed aqueous solution described in Section 2.2 was adjusted to $\mathrm{pH} 5.5$ with dilute $\mathrm{NaOH}$ solution of $0.1 \mathrm{M}$. The resulting solution was considered to be slightly charged positively. The cathode and anode made of aluminum of $15 \mathrm{~mm} \times 60 \mathrm{~mm} \times 0.25 \mathrm{~mm}$ dimensions were dipped in $30 \mathrm{~mL}$ of the solution, and EPD was carried out for $1 \mathrm{~min}$ at $0.1 \mathrm{~V}$ of applied voltage. The separation distance between the electrodes was $10 \mathrm{~mm}$. After EPD, the sample was dried at $50^{\circ} \mathrm{C}$ for $3 \mathrm{~h}$ in air.

2.4. Scanning Electron Microscopy. The samples were coated with amorphous osmium using plasma chemical vapor 


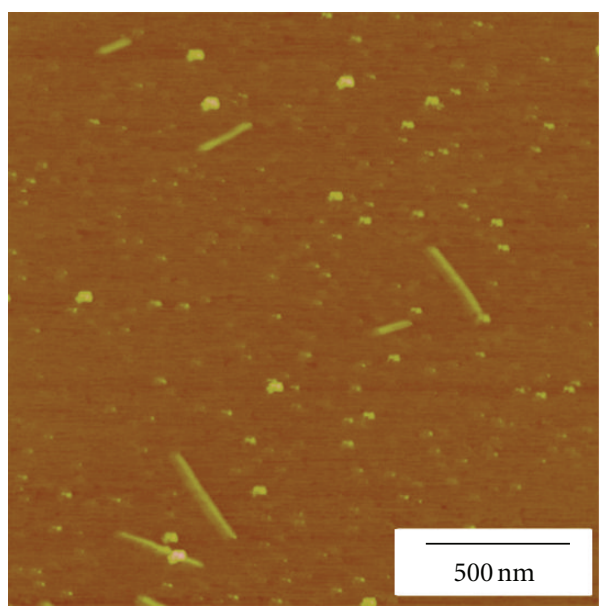

(a)

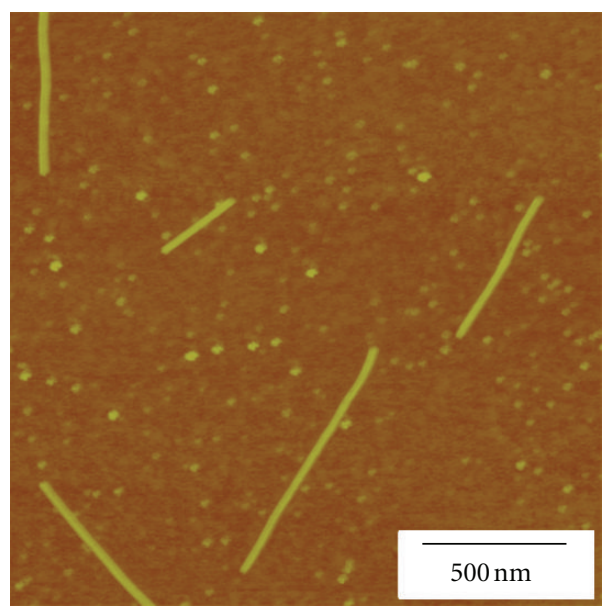

(b)

FIgURE 1: AFM images of products after heating at $95^{\circ} \mathrm{C}$ for (a) $1 \mathrm{~d}$ and (b) $4 \mathrm{~d}$.

deposition (CVD) and then observed morphologically using a field emission scanning electron microscopy (SEM) system (JSM-6301F, JEOL, Japan), with an energy-dispersive spectrometer (EDS).

2.5. Contact Angle Measurement. The static contact angle of water on the fibermats was measured using a CCD camera and SImage mini ver. 5.01 software. The average contact angle was determined from the measurements at ten random points per sample.

2.6. Cell Culture Tests. The fibermats were cut into diskshaped pieces of $15 \mathrm{~mm}$ in diameter with scissors for cell culture tests, and the resulting samples were sterilized using ethylene oxide gas. Murine osteoblast-like cells (MC3T3-E1 cells) were seeded onto the samples in 24 -well plates at a density of 50,000 or 80,000 cells $\cdot$ well $^{-1}$ to evaluate their proliferation or initial adhesion, respectively, and cultured in alpha minimum essential medium ( $\alpha$-MEM) containing $10 \%$ fetal bovine serum (FBS) at $37^{\circ} \mathrm{C}$ in $5 \% \mathrm{CO}_{2}$.

The number of murine osteoblast-like cells (MC3T3-E1 cells) was evaluated using Cell Counting Kit-8 (Dojindo, Japan). The cells were rinsed with $\alpha$-MEM and then incubated in the culture medium containing the reagent of the kit at $37^{\circ} \mathrm{C}$ for $2 \mathrm{~h}$. Cell number was evaluated after measuring the absorbance of the resulting medium at $450 \mathrm{~nm}$. The differences between the samples were determined by Student's $t$-test, with $P<0.05$, which is considered to be statistically significant.

The cells cultured on the samples for $3 \mathrm{~h}$ were fixed in $2.5 \%$ glutaraldehyde for $40 \mathrm{~min}$ at $4^{\circ} \mathrm{C}$, dehydrated through a series of increasing concentrations of ethanol, and finally dried with hexamethyldisilazane. The dried samples were observed by SEM after they were coated with amorphous osmium.

To evaluate the initial adhesion of the cells on the fibers, they were cultured on the samples for $3 \mathrm{~h}$ and then fixed in phosphate buffer solution (PBS) containing 4\% paraformaldehyde for $30 \mathrm{~min}$ at $4^{\circ} \mathrm{C}$. The cells were then treated with PBS containing $1 \%$ bovine serum albumin (BSA) and $0.1 \%$ Triton $\mathrm{X}$ for $25 \mathrm{~min}$ at $4^{\circ} \mathrm{C}$. Finally, they were fluorescence-stained with $50 \mu \mathrm{L}$ of Alexa Fluor 488 Phalloidin (Molecular Probe), which was diluted to a concentration of $2.5 \%$ with $\mathrm{PBS}$, for $30 \mathrm{~min}$ at $37^{\circ} \mathrm{C}$. The samples were observed with a fluorescence microscope (Keyence, BZ-9000).

\section{Results and Discussion}

3.1. Preparation of INT-Coated Fibermats. In our earlier work, PLLA fibermats consisting of skeletal fibers of $\sim 10 \mu \mathrm{m}$ diameter and $\sim 40 \mu \mathrm{m}$ spaces between them, on average, were reported to be suitable for cellular in-growth [28]. Figure 2 shows SEM images of the surface morphologies of the noncoated and INT-coated SiPVH fibermats. Microfibers of $\sim 10 \mu \mathrm{m}$ diameter were observed on the surface and found to be intertwined. The sizes of the spaces between the microfibers varied between $10 \mu \mathrm{m}$ and several hundred micrometers, as shown in Figure 2(a). Numerous pores of submicrometer diameter were found on the surfaces of the fibers, which were due to the volatilization of the solvent of the polymer solution during electrospinning $[29,30]$.

There are almost no significant differences in the appearance between the SiPVH fibermat coated with $\mathrm{INT}_{220}$ and the noncoated one, as shown in Figures 2(a) and 2(b), respectively. On the other hand, in the fibermat coated with $\mathrm{INT}_{570}$ shown in Figure 2(c), weblike plates around cross-linked points of the fibers can be observed.

The EDS spectrum in Figure 2(a) shows the peaks of Ca and $\mathrm{Si}$ due to SiV. The Si peak is small, since the silicon content of SiV is very small $(2.6 \mathrm{wt} \%)$. In the spectra of the INT-coated SiPVH fibermats (Figures 2(b) and 2(c)), $\mathrm{Al}$ peaks are seen in addition to the peaks of $\mathrm{Ca}$ and $\mathrm{Si}$ due to SiV. The Si peak intensities are higher than that in Figure 2(a). The Al contents of the fibermats coated with $\mathrm{INT}_{220}$ and $\mathrm{INT}_{570}$ were estimated to be $\sim 2$ and $\sim 7$ at $\%$, respectively. The element originates from INTs on the surface, since no $\mathrm{Al}$ is included in SiPVH. This indicates 

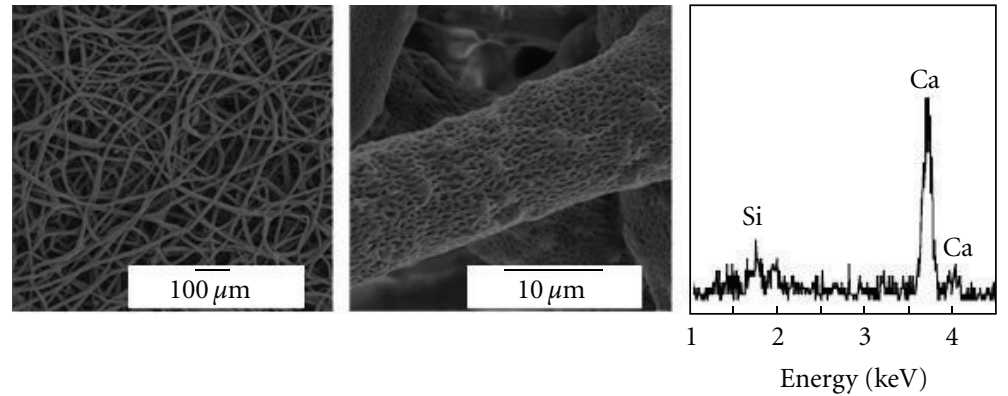

(a)
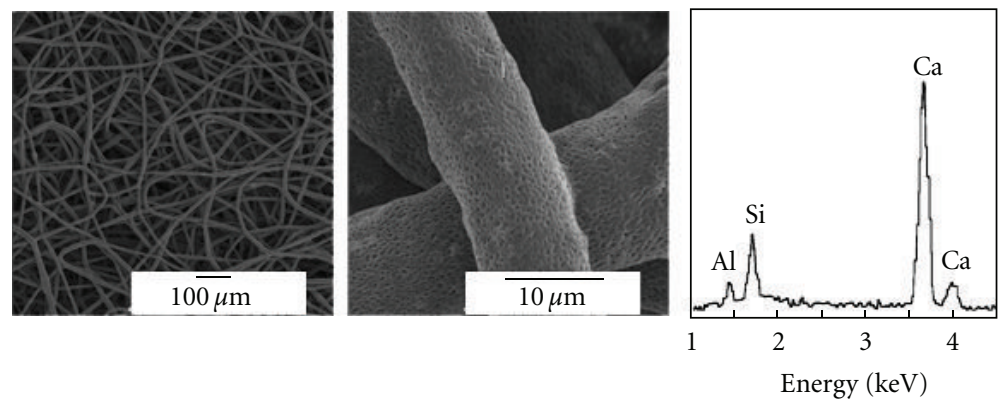

(b)
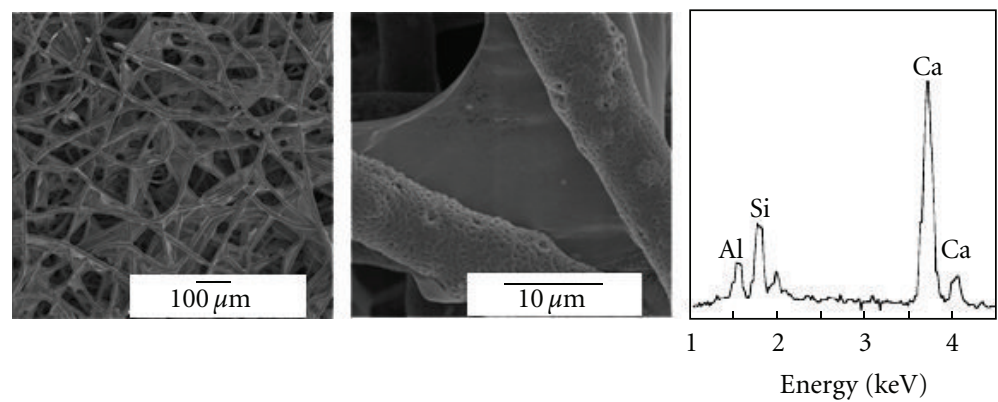

(c)

FIgURE 2: SEM images and EDS spectra of (a) noncoated and (b, c) INT-coated SiPVH fibermats. (b) INT $_{220}$-coated and (c) INT 570 -coated SiPVH fibermats. Center: magnified views; right: EDS spectra.

that INTs were coated on the fibermats by EPD. The weblike plates around cross-linked points, as shown in Figure 2(c), were found to consist of aluminum silicates with a Si/Al ratio of $\sim 0.5$, which corresponds to the INT composition. That is, the plates were concluded to be an aggregation of INTs. It has been reported that the viscosity of the INTdispersed aqueous solution increases with increasing INT length and the number or forming net texture of INTs [24]. INTs attracted to an electrode in an electric field would easily aggregate around the surface of the fibers, since the number of INTs in a dispersed aqueous solution increases. Then, the attracted INTs would likely form bridges around cross-linked points. Since $\mathrm{INT}_{220}$ was shorter than $\mathrm{INT}_{570}$, the incidence possibility of aggregation would be lower.

To discuss the EPD effect, a dip-coating method was used: after the SiPVH fibermat was dipped in the INTdispersed aqueous solution $(0.087 \%)$, it was drawn up at a speed of $\sim 1 \mathrm{~mm} \cdot \mathrm{s}^{-1}$ and then dried. The EDS analysis of the resulting fibermat showed no $\mathrm{Al}$ peak (under the analytical limit). That is, it is difficult to coat the fibermats with INTs by dip coating, and thus EPD is concluded to be a powerful method for such coating. Since the INT concentration in the solution is considerably small, almost no INTs would adhere to the skeletal fibers after the dip coating. It is not easy to prepare the dispersed solution containing a large number of INTs owing to some technical problems, such as impurity formation in the solution, and gelation owing to its concentration. The hydrophobic surface of SiPVH fibers may also be one of the causes of the difficulty in dip coating. In the case of EPD, INTs would pass through the porous structure of fibermats in an electric field and concentrate around the surfaces of skeletal fibers, and then accumulate on them.

The noncoated fibermat exhibited hydrophobicity of a contact angle of $121^{\circ}$. In contrast, a drop of water immediately penetrated the fibermats coated with INTs, which indicated that the hydrophilicity of these fibermats was improved markedly after INT coating. This is because INTs are hydrophilic owing to the hydroxyl groups on their surfaces and have a high water absorbency, attributed to their nanotubular structure. In particular, the fibermat coated 
with $\mathrm{INT}_{220}$ showed excellent hydrophilicity as well as that coated with $\mathrm{INT}_{570}$, although it is difficult to morphologically distinguish them from the noncoated one. A very small number of INTs seem to be sufficient for improving the hydrophilicity of fibermats.

3.2. Cellular Compatibility. The cellular proliferation behavior of the INT-coated fibermats in comparison with that of the noncoated one was evaluated using MC3T3-E1 cells. Figure 3 shows the numbers of cells after culturing on them for 3 days. The numbers of cells after culture on $\mathrm{INT}_{220^{-}}$ and $\mathrm{INT}_{570}$-coated fibermats were significantly higher $(P<$ 0.05 ) than those on the noncoated one on both day 1 and day 3. There were no significant differences in the number between the two types of INT-coated fibermat. This might have been due to the effects of the INT coating, which shows high cellular compatibility [24,25]. Even with coating with a trace amount of $\mathrm{INT}_{220}$, excellent improvement in the compatibility on the fibermat was achieved.

The doubling time (DT) of the number of cells for discussing proliferation behavior is determined by

$$
\mathrm{DT}=\frac{\left(t_{2}-t_{1}\right) \log 2}{\log N_{2}-\log N_{1}},
$$

where $N_{1}$ and $N_{2}$ are the numbers of cells at the culture time points $t_{1}$ and $t_{2}(\mathrm{~h})$, respectively. The DTs of the noncoated, $\mathrm{INT}_{220}$-coated, and $\mathrm{INT}_{570}$-coated fibermats were estimated to be $33.0 \mathrm{~h}, 33.7 \mathrm{~h}$, and $33.7 \mathrm{~h}$, respectively, which are not significantly different. The difference in the numbers of cells after culture between the noncoated and INT-coated fibermats might have originated from the adhesion behavior of the cells at an early stage after seeding.

Figure 4 shows the numbers of cells attached to the noncoated and INT-coated fibermats at $3 \mathrm{~h}$ after seeding. The numbers of cells cultured on the INT-coated fibermats were significantly higher than that on the noncoated one. INTs were suggested to enhance the initial cell attachment to the fibermats. However, there was no significant difference in the number of cells cultured between $\mathrm{INT}_{220}$ and $\mathrm{INT}_{570}$.

Figures 5 and 6 show the morphologies of the cells attached to the fibermats at $3 \mathrm{~h}$ after seeding. The cells adhering to the noncoated fibermat were still spherical and were preferentially attached to the surface around cross-linked points between skeletal fibers, as shown in Figure 5(a). On the other hand, as shown in Figures 5(b) and 5(c), the cells on the INT-coated fibermats were elongated not only on the surface around the cross-linked points but also on individual fibers and actin filaments were aligned to the fibers. The cells were attached over the entire surface of the INT-coated fibermats, as shown in Figures 6(b) and 6(c). These cell culture tests suggested that INTs enhanced cell adhesion and growth.

Watari and coworkers reported that protein adsorption on nanofibrous scaffolds contributes to excellent cell adhesion and growth [21-23]. In our preliminary work, INTs showed a high adsorbability to various types of protein including acidic, neutral, and basic ones, owing to the large specific surface area of the nanotubular structure. Thus, it is proposed that cell-adhesive proteins, such as fibronectin

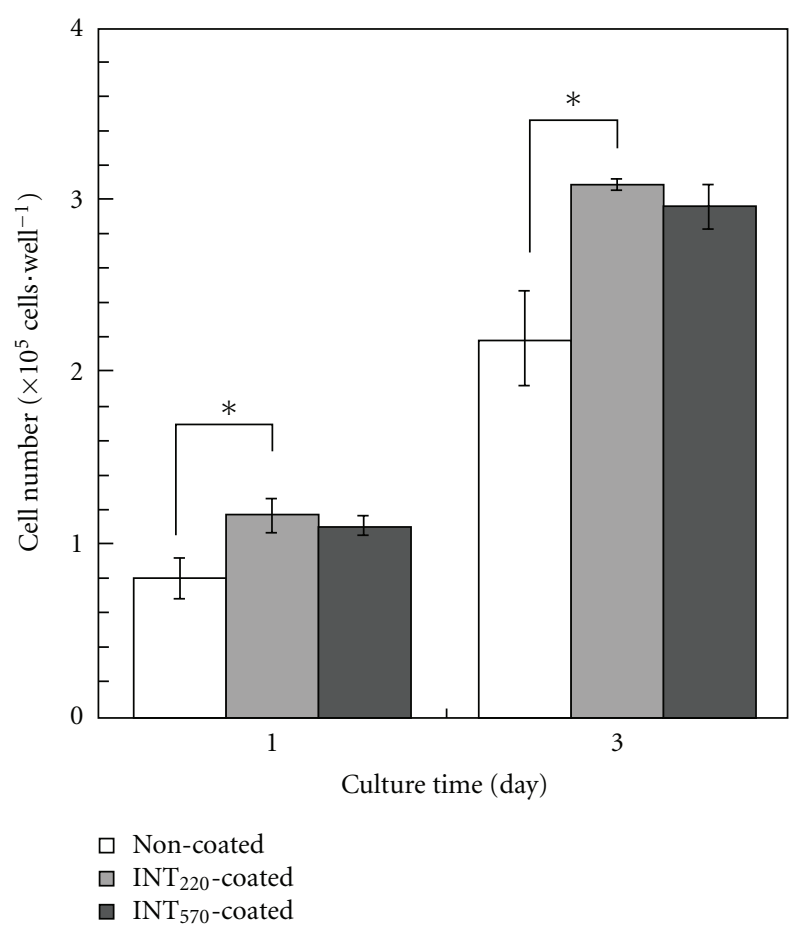

FIGURE 3: Numbers of cells attached to noncoated and INT-coated fibermats after 3 days of culture.

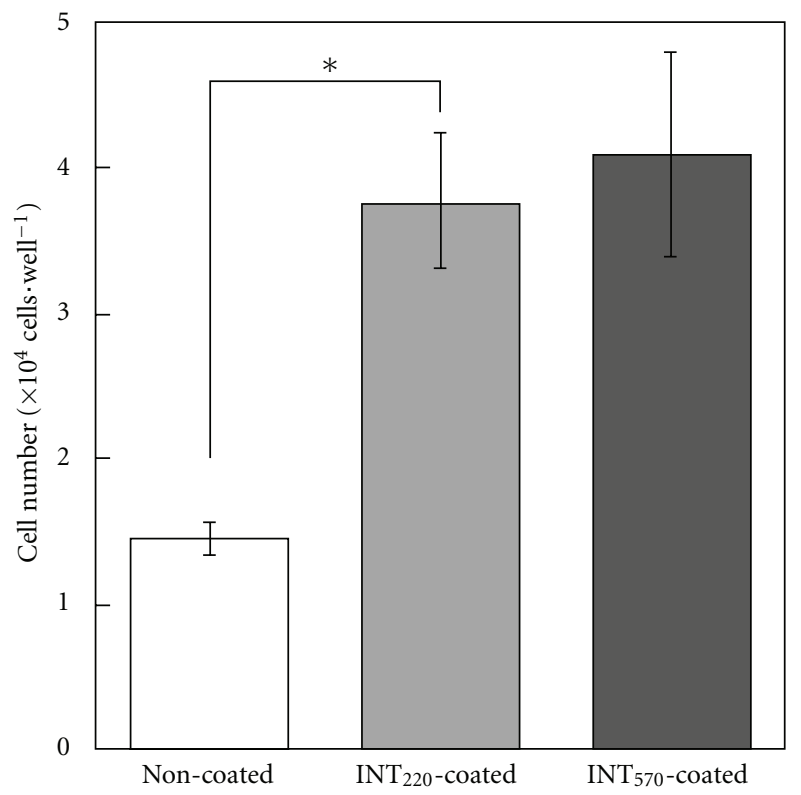

FIGURE 4: Numbers of cells attached to noncoated and INT-coated fibermats at $3 \mathrm{~h}$ after seeding.

and vitronectin, could be easily adsorbed on the INT surface $[31,32]$.

Furthermore, the INT nanostructure may also be related to cell adhesion. Hirata et al. reported that the nanostructure of multiwalled carbon nanotubes might be effective in cell entrapment [33]. The geometrical factor in the INT nanostructure may be beneficial for cell attachment to surfaces. 


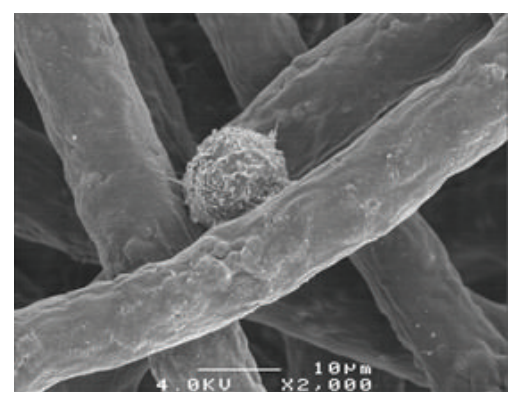

(a)

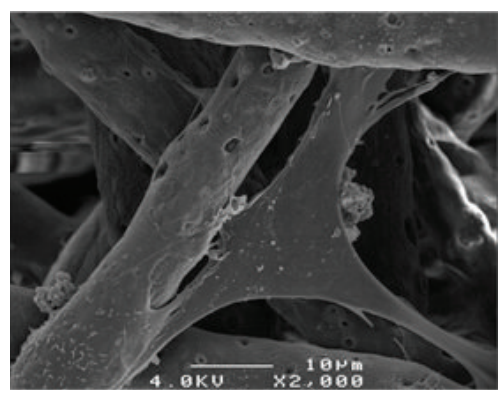

(b)

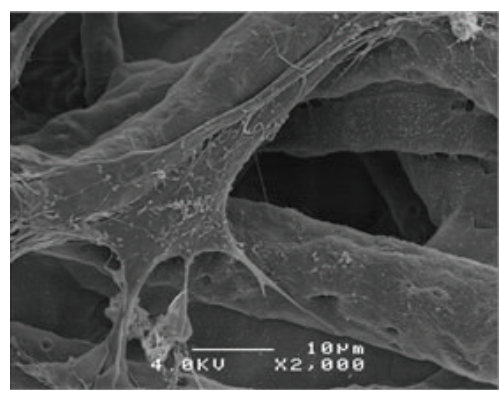

(c)

FIGURE 5: SEM images of cells on (a) noncoated and (b, c) INT-coated SiPVH fibermats at $3 \mathrm{~h}$ after seeding. (b) INT $_{220}$-coated and (c) INT $_{570}$-coated SiPVH fibermats.

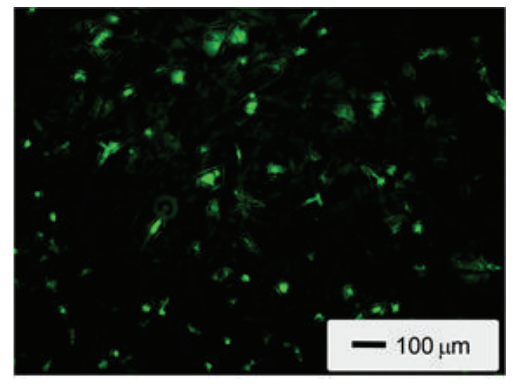

(a)

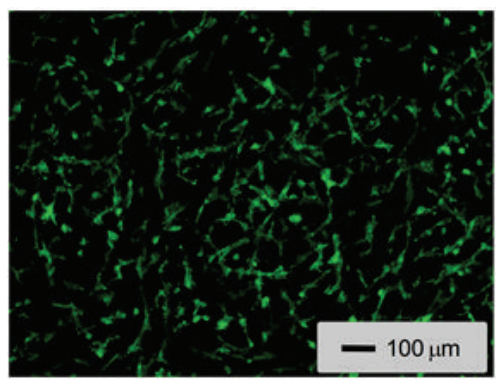

(b)

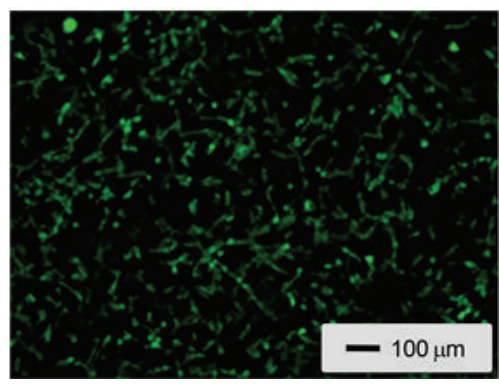

(c)

FIGURE 6: Fluorescence micrographs of cells on (a) noncoated and (b, c) INT-coated SiPVH fibermats at $3 \mathrm{~h}$ after seeding. (b) INT ${ }_{220}$-coated and (c) $\mathrm{INT}_{570}$-coated SiPVH fibermats.

In the present work, a trace amount of INT was coated on SiPVH fibers: no existence could be observed by SEM. The measurement of the INT amount on the fibers is not easy and the investigation is in progress. Although there might be almost no need to consider the influence of the $\mathrm{Al}^{3+}$ ion dissolved from the trace amount of INT on living body, we would have to discuss its behavior in future.

There are almost no reports on the solubility of aluminum silicates in living body or under its simulated environment, to the best of our knowledge. Although imogolite has been reported to dissolve slightly in alkaline solutions of $\mathrm{pH}>10$ [34] and in acid solutions of $\mathrm{pH}<3$ [35], almost no information around the neutrality is seen. It would be important to examine the solubility of INTs in detail.

\section{Conclusion}

Aluminum silicate INTs were coated successfully on an electrospun fibermat, consisting of poly(L-lactic acid)-based composite microfibers containing siloxane-doped calcium carbonate particles by electrophoretic deposition using an applied voltage of $0.1 \mathrm{~V}$ for $1 \mathrm{~min}$. INTs of 220 and $570 \mathrm{~nm}$ lengths on average were coated very thinly such that their presence could not be observed by SEM. In the case of coating with INTs of $570 \mathrm{~nm}$ length, weblike plates formed around cross-linked points of skeletal fibers owing to their aggregation. Shorter INTs were better for preparing a homogeneous coating on a fibermat. The hydrophilicity of the fibermat was improved markedly after INT coating. Cell culture tests indicated that INT enhanced the attachment and extension of MC3T3-E1 cells. The excellent compatibility might have originated from the protein adsorbability and geometrical factor of the nanostructure. Thus INT coating may be one of the best approaches to improving the hydrophilicity and cellular compatibility of biomaterials for bone regeneration.

\section{Acknowledgments}

The authors are indebted to Dr. Yoshio Ota, Dr. Xianfeng Yao, and Dr. Kazuya Oribe of Orthorebirth Co., Ltd., for their helpful discussions. This work was supported in part by Adaptable and Seamless Technology Transfer Program through Target-driven R\&D (A-STEP; Seeds actualization type: AS2311416F) from Japan Science and Technology Agency.

\section{References}

[1] S.-S. Kim, M. S. Park, O. Jeon, C.-Y. Choi, and B. S. Kim, "Poly(lactide-coglycolide)/hydroxyl-apatite composite scaffolds for bone tissue engineering," Biomaterials, vol. 27, no. 8, pp. 1399-1409, 2006.

[2] K. Zhang, Y. Wang, M. A. Hillmyer, and L. F. Francis, "Processing and properties of porous poly(L-lactic)/bioactive glass composites," Biomaterials, vol. 25, no. 13, pp. 2489-2500, 2004.

[3] L. G. Griffith, "Polymeric biomaterials," Acta Materialia, vol. 48, no. 1, pp. 263-277, 2000. 
[4] R. Zhang and P. X. Ma, "Porous poly(L-lactic acid)/apatite composites created by biomimetic process," Journal of Biomedical Materials Research, vol. 45, no. 4, pp. 285-293, 1999.

[5] J. A. Roether, A. R. Boccaccini, L. L. Hench, V. Maquet, S. Gautier, and R. Jérôme, "Development and in vitro characterisation of novel bioresorbable and bioactive composite materials based on polylactide foams and Bioglass for tissue engineering applications," Biomaterials, vol. 23, no. 18, pp. 3871-3878, 2002.

[6] I. D. Xynos, A. J. Edgar, L. D. K. Buttery, L. L. Hench, and J. M. Polak, "Ionic products of bioactive glass dissolution increase proliferation of human osteoblasts and induce insulin-like growth factor II mRNA expression and protein synthesis," Biochemical and Biophysical Research Communications, vol. 276, no. 2, pp. 461-465, 2000.

[7] D. M. Reffitt, N. Ogston, R. Jugdaohsingh et al., "Orthosilicic acid stimulates collagen type 1 synthesis and osteoblastic differentiation in human osteoblast-like cells in vitro," Bone, vol. 32, no. 2, pp. 127-135, 2003.

[8] N. Patel, S. M. Best, W. Bonfield et al., "A comparative study on the in vivo behavior of hydroxyapatite and silicon substituted hydroxyapatite granules," Journal of Materials Science, vol. 13, no. 12, pp. 1199-1206, 2002.

[9] A. Obata, S. Tokuda, and T. Kasuga, "Enhanced in vitro cell activity on silicon-doped vaterite/poly(lactic acid) composites," Acta Biomaterialia, vol. 5, no. 1, pp. 57-62, 2009.

[10] T. J. Still and H. A. von Recum, "Electrospinning: applications in drug delivery and tissue engineering," Biomaterials, vol. 29, no. 13, pp. 1989-2006, 2008.

[11] W. J. Li, C. T. Laurencin, E. J. Caterson, R. S. Tuan, and F. K. Ko, "Electrospun nanofibrous structure: a novel scaffold for tissue engineering," Journal of Biomedical Materials Research, vol. 60, no. 4, pp. 613-621, 2002.

[12] X. Xin, M. Hussain, and J. J. Mao, "Continuing differentiation of human mesenchymal stem cells and induced chondrogenic and osteogenic lineages in electrospun PLGA nanofiber scaffold," Biomaterials, vol. 28, no. 2, pp. 316-325, 2007.

[13] Y. Z. Zhang, B. Su, J. Venugopal, S. Ramakrishna, and C. T. Lim, "Biomimetic and bioactive nanofibrous scaffolds from electrospun composite nanofibers," International Journal of Nanomedicine, vol. 2, no. 4, pp. 623-638, 2007.

[14] A. O. Brightman, B. P. Rajwa, J. E. Sturgis, M. E. McCallister, J. P. Robinson, and S. L. Voytik-Harbin, "Timelapse confocal reflection microscopy of collagen fibrillogenesis and extracellular matrix assembly in vitro," Biopolymers, vol. 54, no. 3, pp. 222-234, 2000.

[15] A. S. Badami, M. R. Kreke, M. S. Thompson, J. S. Riffle, and A. S. Goldstein, "Effect of fiber diameter on spreading, proliferation, and differentiation of osteoblastic cells on electrospun poly(lactic acid) substrates," Biomaterials, vol. 27, no. 4, pp. 596-606, 2006.

[16] M. M. Stevens and J. H. George, "Exploring and engineering the cell surface interface," Science, vol. 310, no. 18, pp. 1135$1138,2005$.

[17] J.-H. Jang, O. Castano, and H. W. Kim, "Electrospun materials as potential platforms for bone tissue engineering," Advanced Drug Delivery Reviews, vol. 61, no. 12, pp. 1065-1083, 2009.

[18] A. Obata, T. Hotta, T. Wakita, Y. Ota, and T. Kasuga, "Electrospun microfiber meshes of silicon-doped vaterite/poly(lactic acid) hybrid for guided bone regeneration," Acta Biomaterialia, vol. 6, no. 4, pp. 1248-1257, 2010.

[19] T. Wakita, A. Obata, G. Poologasundarampillai, J. R. Jones, and T. Kasuga, "Preparation of electrospun siloxane-poly(lactic acid)-vaterite hybrid fibrous membranes for guided bone regeneration," Composites Science and Technology, vol. 70, no. 13, pp. 1889-1893, 2010.

[20] P. D. G. Cradwick, V. C. Farmer, J. D. Russell, C. R. Masson, K. Wada, and N. Yoshinaga, "Imogolite, a hydrated aluminum silicate of tubular structure," Nature Physical Science, vol. 240, no. 104, pp. 187-189, 1972.

[21] F. Watari, S. Abe, C. Koyama et al., "Behavior of in vitro, in vivo and internal motion of micro/nano particles of titanium, titanium oxides and others," Journal of the Ceramic Society of Japan, vol. 116, no. 1349, pp. 1-5, 2008.

[22] F. Watari, N. Takashi, A. Yokoyama et al., "Material nanosizing effect on living organisms: non-specific, biointeractive, physical size effects," Journal of the Royal Society Interface, vol. 6, no. 3, pp. S371-S388, 2009.

[23] F. Watari, "Biointeractive and bioreactive nature of nanomaterials," Nano Biomedicine, vol. 1, no. 1, pp. 2-8, 2009.

[24] K. Ishikawa, T. Akasaka, Y. Nodasaka et al., "Physical properties of aluminosilicate nanotubes, imogolite, as scaffold and effect on osteoblastic mineralization," Nano Biomedicine, vol. 1, no. 2, pp. 109-120, 2009.

[25] K. Ishikawa, S. Abe, Y. Yawaka, M. Suzuki, and F. Watari, "Osteoblastic cellular responses to aluminosilicate nanotubes, imogolite using Saos-2 and MC3T3-E1 cells," Journal of the Ceramic Society of Japan, vol. 118, no. 1378, pp. 516-520, 2010.

[26] M. Suzuki, H. Sato, C. Ikeda, R. Nakanishi, K. Inukai, and M. Maeda, "State change of imogolite according to heating duration on synthesizing," Journal of Clay Science Society of Japan, vol. 46, no. 4, pp. 194-199, 2007 (Japanese).

[27] H. Tsuchida, S. Ooi, K. Nakaishi, and Y. Adachi, "Effects of pH and ionic strength on electrokinetic properties of imogolite," Colloids and Surfaces A, vol. 265, no. 1-3, pp. 131-134, 2005.

[28] K. Fujikura, A. Obata, and T. Kasuga, "Cellular migration to electrospun poly(lactic acid) fibermats," Journal of Biomaterials Science, vol. 23, pp. 1939-1950, 2012.

[29] Z. M. Huang, Y. Z. Zhang, M. Kotaki, and S. Ramakrishna, "A review on polymer nanofibers by electrospinning and their applications in nanocomposites," Composites Science and Technology, vol. 63, no. 15, pp. 2223-2253, 2003.

[30] G.-T. Kim, J.-S. Lee, J.-H. Shin et al., "Investigation of pore formation for polystyrene electrospun fiber: effect of relative humidity," Korean Journal of Chemical Engineering, vol. 22, no. 5, pp. 783-788, 2005.

[31] J. G. Steele, G. Johnson, and P. A. Underwood, "Role of serum vitronectin and fibronectin in adhesion of fibroblasts following seeding onto tissue culture polystyrene," Journal of Biomedical Materials Research, vol. 26, no. 7, pp. 861-884, 1992.

[32] T. A. Horbett and M. B. Schway, "Correlations between mouse 3T3 cell spreading and serum fibronectin adsorption on glass and hydroxyethylmethacrylate-ethylmethacrylate copolymers," Journal of Biomedical Materials Research, vol. 22, no. 9, pp. 763-793, 1988.

[33] E. Hirata, M. Uo, Y. Nodasaka et al., "3D collagen scaffolds coated with multiwalled carbon nanotubes: initial cell attachment to internal surface," Journal of Biomedical Materials Research B, vol. 93, no. 2, pp. 544-550, 2010.

[34] Z. Abidin, N. Matsue, and T. Henmi, "Dissolution mechanism of nano-ball allophone with dilute alkali solution," Clay Science, vol. 12, no. 4, pp. 213-222, 2004.

[35] C. Su, J. B. Harsh, and J. S. Boyle, "Solubility of hydroxy-aluminum interlayers and imogolite in a Spodosol," Soil Science Society of America Journal, vol. 59, no. 2, pp. 373-379, 1995. 

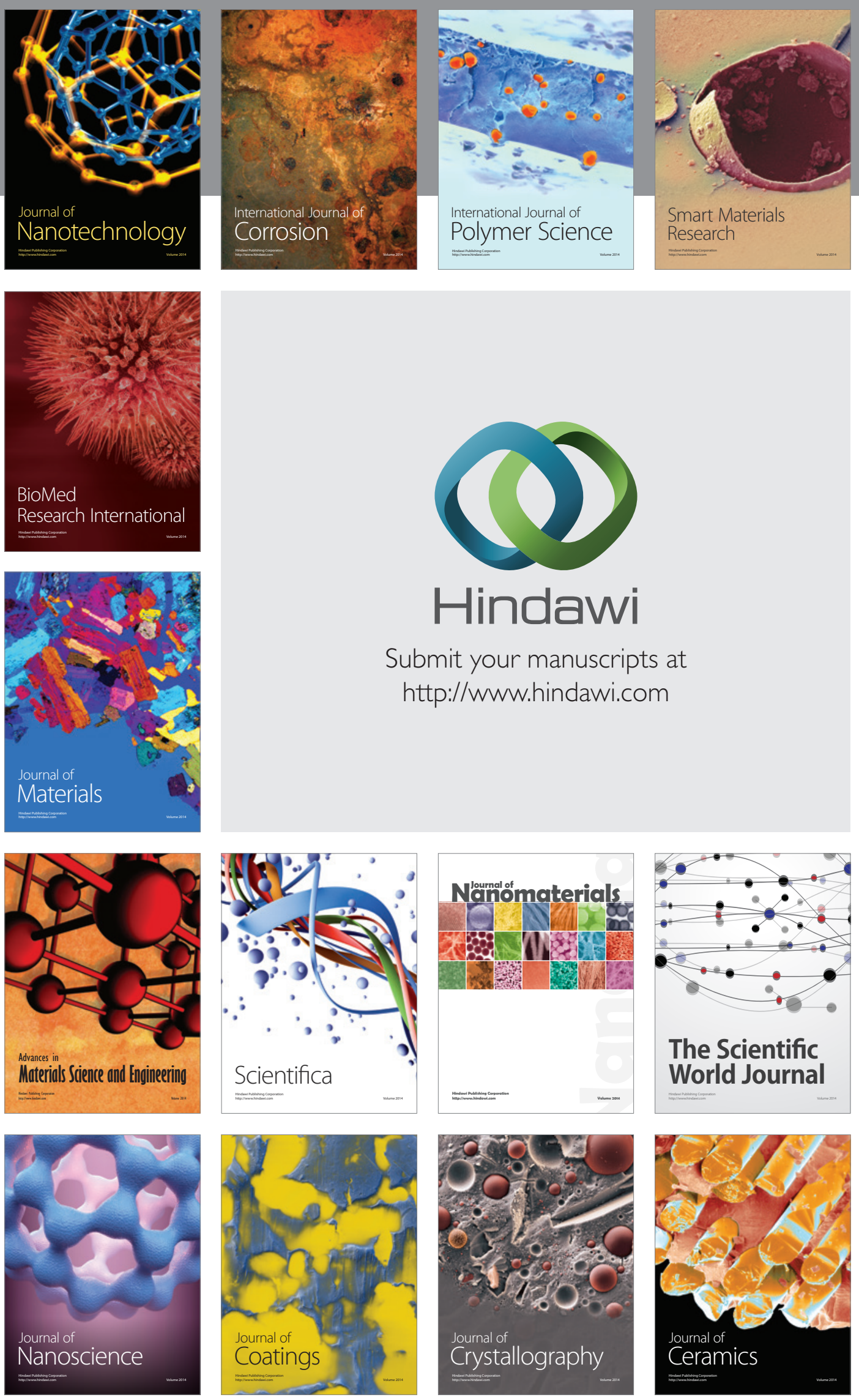

The Scientific World Journal

Submit your manuscripts at

http://www.hindawi.com

\section{World Journal}

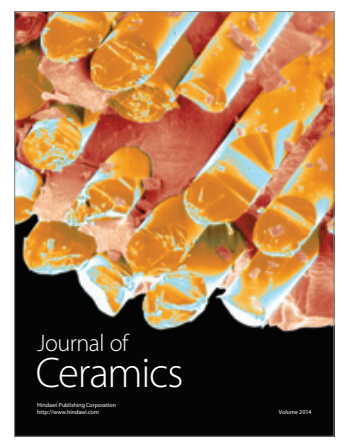

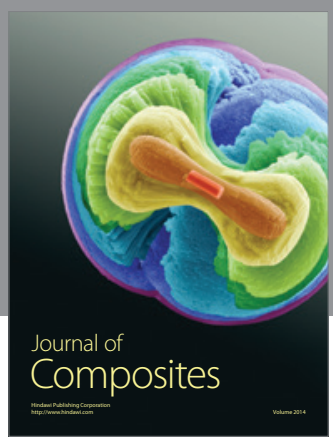
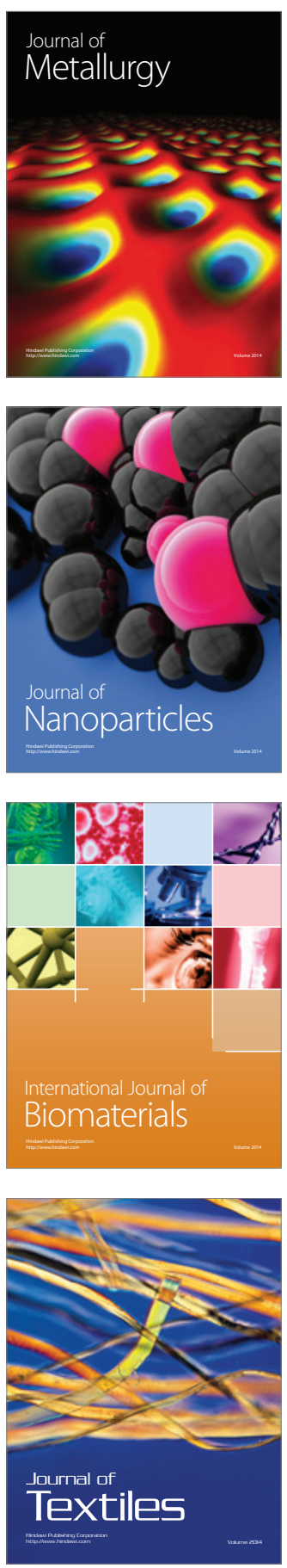